\title{
Safety of ERCP in patients with liver cirrhosis: a national database study
}

\section{(잉 $\circledast$}

\author{
Authors \\ Udayakumar Navaneethan ${ }^{1}$, Basile Njei ${ }^{2}$, Xiang Zhu ${ }^{1}$, \\ Kiran Kommaraju ${ }^{1}$, Mansour A. Parsi ${ }^{3}$, Shyam Varadarajulu ${ }^{1}$ \\ Institutions \\ 1 Center for Interventional Endoscopy, Orlando, FL, USA \\ 2 Department of Gastroenterology, Yale University, CT, USA \\ 3 The Cleveland Clinic, Cleveland, OH, USA
}

submitted 18.5.2016

accepted after revision 17.1.2017

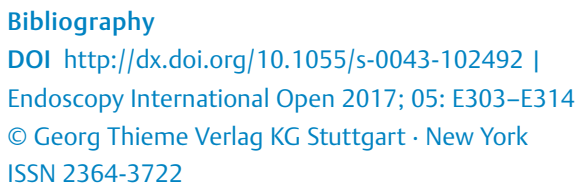

Corresponding author

Udayakumar Navaneethan, MD, Center for Interventional

Endoscopy, University of Central Florida College of Medicine, Florida

Hospital, 601 E Rollins Street, Orlando, FL 32803, USA

Fax: +1-216-444-6305

udhaykumar81@gmail.com

\begin{abstract}
Background and aims Given the limited data on the safety of endoscopic retrograde cholangiopancreatography (ERCP) in patients with liver cirrhosis, we attempted to evaluate this question using a large national database.

Methods We conducted a matched case-control study using the 2010 National Inpatient Sample database in which four non-cirrhotic controls were matched randomly for every cirrhotic patient from the same 10-year age group. We compared adverse events and safety of inpatient ERCP between patients with $(n=3228)$ and without liver cirrhosis (controls, $\mathrm{n}=12$ 912).

Results Of the 3228 cirrhotic patients, 2603 (80.6\%) had decompensated and 625 (19.4\%) had compensated disease. Post-procedure bleeding $(2.1 \%$ vs. $1.2 \%, P<0.01)$ was higher in patients compared to controls. On multivariable analysis, decompensated cirrhosis (adjusted odds ratio [aOR], 2.7; 95\% confidence interval [CI], 2.2 -3.2), compensated cirrhosis ( $\mathrm{OOR} 2.2$; $95 \% \mathrm{Cl} 1.2-3.9$ ), therapeutic ERCPs (aOR 1.4; $95 \% \mathrm{Cl} 1.2-2.1$ ), and biliary sphincterotomy (aOR 1.6; $95 \% \mathrm{Cl} 1.1-2.1$ ) were independently associated with increased risk of post-procedure bleeding. Performing ERCPs in large (aOR 0.5; 95\%Cl 0.4-0.6) and medium (aOR 0.7; 95\%Cl 0.6-0.9) sized hospitals was associated with a decreased risk of post-procedure bleeding. Biliary sphincterotomy (aOR 1.7; $95 \% \mathrm{Cl} 1.2-2.3$ ) and therapeutic ERCPs (aOR 1.1; $95 \% \mathrm{Cl} 1.1-1.3$ ) increased the risk of post-ERCP pancreatitis, and pancreatic stent placement was associated with a decreased risk of post-ERCP pancreatitis (aOR $0.8 ; 95 \% \mathrm{Cl} 0.7-0.9)$.

Conclusions Cirrhosis (both compensated and decompensated), performing therapeutic ERCPs and biliary sphincterotomy increase the risk of post-procedure bleeding. Performing ERCPs in large and medium sized hospitals may improve outcomes.
\end{abstract}

\section{Introduction}

Endoscopic retrograde cholangiopancreatography (ERCP) is the treatment modality of choice for pancreatobiliary disorders with more than half a million procedures being performed annually [1]. Patients with underlying liver cirrhosis may develop common bile duct stones that may require ERCP. They are also at an increased risk of adverse events due to underlying liver dysfunction [2,3]. It is well known that advanced liver disease is a significant risk factor for perioperative complications after surgery [4]. The safety and outcomes of ERCP in cirrhotic patients is unclear as the literature for safety of ERCP in cirrhosis has consisted mainly of small retrospective studies $[3,5,6]$.

Although a retrospective study reported that there was no increased risk of adverse events in cirrhotic patients undergoing ERCP, patients with Child-Pugh (CP) Class B and $C$ had a higher rate of adverse events compared to CP Class A [5]. Other studies have suggested that the risk of ERCP-related bleeding is increased in cirrhotic patients [3,6]. Given the lack of popula- tion-based studies and conflicting literature on the safety of ERCP in cirrhotic patients, we attempted to address this question using a national database.

The aims of our study were (1) to assess the prevalence of various ERCP-related adverse events in patients with cirrhosis, (2) to compare rates of adverse events with non-cirrhotic controls, and (3) to evaluate the impact of cirrhosis on adverse events, length of stay, and hospital costs.

\section{Methods}

\section{Data source}

We obtained data from the Nationwide Inpatient Sample (NIS) database which is the largest all-payer inpatient care database in the US. The NIS database is developed for the Healthcare Cost and Utilization Project (HCUP) and it represents about $20 \%$ of the stratified sample of US community hospitals, including all non-federal general and subspecialty hospitals, public hospitals and academic medical centers. The data includes demographic 
variables (including age, gender, race/ethnicity), discharge disposition, primary and secondary diagnoses (up to 15), primary and secondary procedures (up to 15), primary insurance payers, total hospital charges, and length of stay. For our analysis, we used data from the HCUP NIS for the year 2010, which contains data from 45 states and 1051 hospitals, accounting for over 8 million discharges.

\section{Study groups, definitions, inclusion and exclusion criteria}

Using the 2010 NIS dataset, all patients between the ages of 18 and 90 years at admission and who underwent an inpatient ERCP were included in the study. The patient population and controls were selected based on procedural coding in accordance with the International Classification of Diseases, 9th revision, Clinical Modification (ICD-9 CM).

We classified the indications for ERCP into purely diagnostic ERCP and therapeutic ERCP. Indications for ERCP were further classified as biliary and pancreatic indications. The ICD-9 CM procedure codes are included in Appendix 1.

The control population was selected among non-cirrhotic patients who underwent ERCP by using stratified sampling. The controls were selected randomly using the "radmatch" code in the Stata 13.0 software package (Stata Corp LP, College Station, TX, United States) in which four non-cirrhotic controls were matched randomly for every cirrhotic patient from the same 10-year age group. The sampling design is shown in - Fig. 1 .

The study group included patients with a concomitant diagnosis of cirrhosis (ICD-9 CM codes 571.2, 571.5, 571.6; Appendix 1). Patients with coexisting ICD-9-CM codes for variceal hemorrhage were included in the initial analysis as long as it was not a primary diagnosis code. The study group was further classified into decompensated and compensated cirrhosis. We used previously defined criteria for compensated and decompensated liver disease using the Baveno IV classification of cirrhosis severity [7]. The patients with compensated liver disease were defined as those without ascites, variceal bleeding or hepatic encephalopathy: Baveno IV stages 1 and 2 . The patients with decompensated liver disease were defined as those with a concomitant diagnosis of diagnosis of variceal bleed (ICD 9 code 456.0, 456.2), ascites (789.5), portal hypertension (572.3) or hepatic encephalopathy (572.2) (Baveno IV stages 3 and 4) [7].

We classified hospitals into small, medium and large based on bed size which was defined based on region of the United States, the urban-rural designation of the hospital, and the teaching status in the NIS database. Appendix 2 describes how the bed size is defined based on the NIS database. In addition to the demographic information (age, race, and sex), health insurance status was derived, classified as private, Medicare, Medicaid, or other/unknown.

\section{Outcomes}

The primary outcomes evaluated were ERCP-related adverse events (AEs) (pancreatitis, bleeding, infection, perforation, and mortality). The secondary outcomes included length of

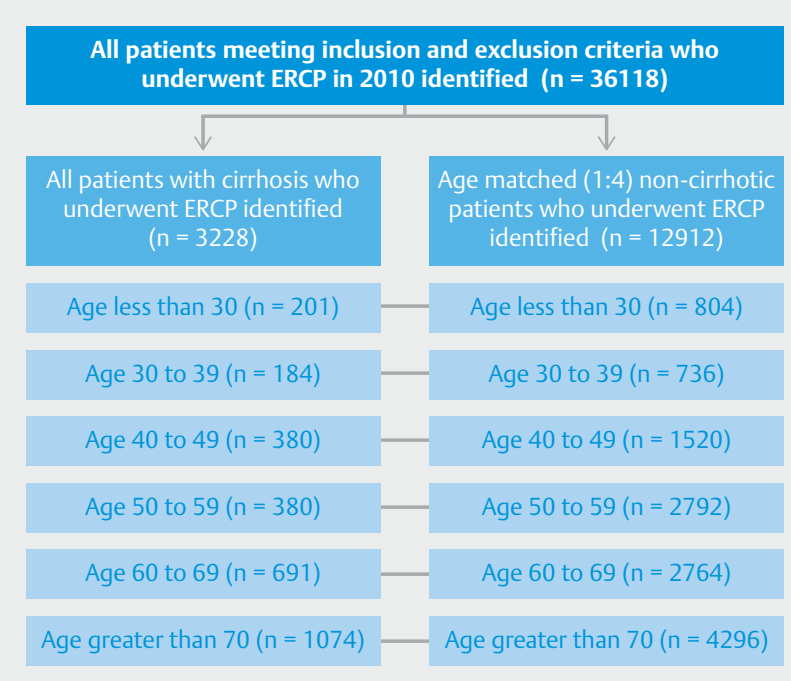

- Fig. 1 Selection of cirrhotic patients and controls in the National Inpatient Sample database. The controls were selected in an agematched fashion at a 1:4 ratio.

stay and hospital costs. We adopted the previously defined definition for post-ERCP pancreatitis (PEP) from the NIS database that has been validated before $[8,9]$. PEP requires a billing code of ICD-9 code 997.4 (complication of gastrointestinal procedure) along with the pancreatitis code, and previous studies have validated the diagnosis of PEP based on this [8, 9]. Bleeding after ERCP was identified by specific ICD-9 codes used to define post-ERCP hemorrhage (998.11, 909.3, and V58.89). Cholecystitis after ERCP was identified by using ICD-9 codes 575.0 and 575.1. Perforation after ERCP was identified by using ICD-9 code 569.83 .

\section{Comorbidities}

Comorbid conditions were recorded using the Elixhauser comorbidity algorithm, which is a commonly used risk adjustment measure [10]. We used this instead of the Charlson comorbidity score [11] because Elixhauser has been suggested to be a superior risk-adjustment model in patients with cardiac and respiratory conditions commonly seen in patients with cirrhosis [12].

The NIS database consists of deidentified data with no risk of loss of confidentiality. The present study was exempt from Florida Hospital Institutional Review Board review. The data user agreement was completed with the Agency for Healthcare Research and Quality before using the NIS database.

\section{Statistical analysis}

All statistical analyses were performed using the Stata 13.0 software package (Stata Corp LP, College Station, TX, United States) to adjust for the complex sampling design of the NIS. Hospital and discharge level weights were applied to the NIS 2010 data as appropriate for the analysis.

Statistical analysis was performed using chi-squared tests for categorical data and the Student's $t$ test for continuous 
data. A $P$ value of less than 0.05 was considered statistically significant. Bivariable analyses were performed to assess the indications and adverse events of ERCP in cirrhosis. We also used multivariable logistic regression to assess the association between cirrhosis and PEP and post-ERCP bleeding while adjusting for age, gender, race/ethnicity, health insurance, comorbidity, teaching hospital status, diagnostic vs therapeutic indication for ERCP, biliary vs pancreatic indications for ERCP, alcoholic vs non-alcoholic cirrhosis, ERCP related interventions such as sphincterotomy and pancreatic stent placement, and the hospital bed size. Total hospital costs were also obtained. The charge information represents the amount that hospitals billed for services. The national cost estimates were determined by multiplying total charges by a hospital-wide, cost-to-charge ratio per hospital derived from the Centers for Medicare and Medicaid standardized hospital accounting reports.

\section{Results}

Of 7800441 discharges recorded in the NIS 2010 database, we found a total of 3228 discharges for cirrhotic patients who underwent ERCP. Age-matched controls were selected in a 1:4 ratio (controls, $n=12$ 912). Among the 3228 patients with cirrhosis, 2603 (80.6\%) had decompensated and 625 (19.4\%) had compensated disease.

- Table 1 presents the characteristics of the two groups. Patients in the cirrhotic group were more likely to be males with Medicare insurance. Patients in the cirrhotic group had less comorbidity as determined by Elixhauser index. There was no difference between the two groups with respect to pre-procedure sepsis, admission to a teaching hospital, weekend admission, and rural/urban location.

- Table 1 Patient characteristics of cirrhotic inpatients and non-cirrhotic controls who underwent ERCP.

\begin{tabular}{|c|c|c|c|}
\hline & Cirrhosis $(n=3228)$ & No cirrhosis ( $n=12912$ ) & $P$ value \\
\hline Age, mean $(95 \% \mathrm{Cl})$, years & $61.6(61.1-62.3)$ & $61.2(61.0-62.1)$ & 0.53 \\
\hline \multicolumn{4}{|l|}{ Sex } \\
\hline - Female & 46.4 & 61.5 & 0.01 \\
\hline - Male & 53.6 & 38.5 & \\
\hline \multicolumn{4}{|l|}{ Race/ethnicity, \% } \\
\hline - White & 70.0 & 67.7 & 0.25 \\
\hline - Black & 10.8 & 9.6 & \\
\hline - Hispanic & 14.5 & 16.0 & \\
\hline - Other & 4.7 & 6.7 & \\
\hline Weekend admission, \% & 22.9 & 22.3 & 0.47 \\
\hline Elective admission, \% & 8.9 & 9.3 & 0.52 \\
\hline \multicolumn{4}{|l|}{ Primary payer, \% } \\
\hline - Medicare & 47.2 & 43.0 & 0.01 \\
\hline - Medicaid & 13.8 & 13.0 & \\
\hline - Private & 28.2 & 33.1 & \\
\hline - Other & 10.8 & 10.9 & \\
\hline \multicolumn{4}{|l|}{ Median zip code income, \% } \\
\hline - 1st quartile (\$1 - 40 999) & 27.8 & 26.1 & 0.08 \\
\hline - 2nd quartile (\$41 000 -50 999) & 25.6 & 25.4 & \\
\hline - 3 rd quartile (\$51 000 - 66 999) & 25.3 & 25.3 & \\
\hline - 4 th quartile (> \$67 000) & 21.3 & 23.2 & \\
\hline \multicolumn{4}{|l|}{ Elixhauser index, \% } \\
\hline .0 & 58.1 & 51.6 & 0.02 \\
\hline . 1 & 23.2 & 26.5 & \\
\hline .2 & 10.5 & 11.1 & \\
\hline . $\geq 3$ & 8.2 & 10.8 & \\
\hline
\end{tabular}


- Table 1 (Continuation)

\begin{tabular}{|c|c|c|c|}
\hline & Cirrhosis $(n=3228)$ & No cirrhosis $(n=12912)$ & $P$ value \\
\hline Sepsis, \% & 2.1 & 1.9 & 0.73 \\
\hline \multicolumn{4}{|l|}{ Hospital size, \% } \\
\hline - Small & 8.1 & 11.5 & 0.06 \\
\hline - Medium & 22.6 & 25.6 & \\
\hline - Large & 69.3 & 62.9 & \\
\hline \multicolumn{4}{|l|}{ Hospital region, \% } \\
\hline - Northeast & 12.7 & 14.6 & 0.01 \\
\hline - Midwest & 17.9 & 21.0 & \\
\hline - South & 39.0 & 39.4 & \\
\hline - West & 30.4 & 25.0 & \\
\hline Teaching hospital, \% & 51.7 & 50.9 & 0.62 \\
\hline Rural (vs urban) location, \% & 6.0 & 6.4 & 0.11 \\
\hline
\end{tabular}

Cirrhotic patients who underwent ERCP had an increased length of stay compared with controls (mean, 9.1 vs. 6.5 days, $P<0.01)$. The total cost of hospitalization was higher for cirrhotics than non-cirrhotic patients (\$79 282 [631.2] vs. \$57 786 [612.9], mean [SE], $P<0.01)$.

\section{Indications for ERCP}

Common bile duct stones with or without gallstones was the most common indication for ERCP among cirrhotics. > Table 2 summarizes the indications for ERCP in patients with cirrhosis and controls. Approximately $18.9 \%$ of the patients in the cirrhosis group had pre-procedure cholangitis. Patients with cirrhosis were significantly less likely to have ERCP performed for acute biliary pancreatitis ( $26.6 \%$ vs. $33.8 \%, P<0.01)$. There were 165 patients with chronic pancreatitis in the cirrhosis group (5.1\%) and 611 patients in the non-cirrhotic group (4.7\%). There was no statistically significant difference in the proportion of chronic pancreatitis between the two groups ( $P$ $=0.37$ ).

\section{Adverse events}

On bivariable analysis, ERCP-associated adverse events of pancreatitis ( $12 \%$ vs. $10.4 \%, P=0.01)$ and bleeding $(2.1 \%$ vs. $1.2 \%$, $P<0.01)$ were higher in patients with cirrhosis compared to controls. There was no statistically significant difference detected in perforation $(0.2 \%$ vs. $0.1 \%, P=0.8)$ or infection $(0.3 \%$ vs. $0.4 \%, P=0.5$ ) between patients with and without cirrhosis. Additionally, patients with cirrhosis undergoing ERCP were less likely to have a pancreatic stent placed compared to non-cirrhotics ( $5.5 \%$ vs. $6.9 \%, P=0.01$ ). Cirrhotic patients undergoing ERCP were less likely to have biliary sphincterotomy or papillotomy performed compared to those without cirrhosis (57.8\% vs. $68.6 \%, P<0.01)$. Supplementary Table 1 demonstrates the risk of adverse events in alcohol and non-alcohol related cirrhosis. The risk of post-ERCP pancreatitis (PEP) was significantly higher in patients with alcoholic cirrhosis compared to non-alcoholic cirrhotic patients. Alcoholic cirrhotic patients who underwent ERCP also had increased length of hospital stay and incurred higher hospitalization costs.

- Table 3 summarizes the adverse events of ERCP in patients with and without cirrhosis. - Table 4 summarizes the adverse events of ERCP in patients for diagnostic and therapeutic indications and for biliary and pancreatic indications in patients with and without cirrhosis. There was no significant difference in the risk of adverse events in patients with and without cirrhosis who underwent ERCP for purely diagnostic indications or for pancreatic indications.

\section{Multivariable analysis of post-ERCP pancreatitis (PEP)}

On multivariable analysis, the risk of developing PEP with compensated cirrhosis (1.0; $95 \%$ confidence interval $(\mathrm{Cl}) 0.6-1.2$ ) was not statistically significantly different from the risk of developing PEP with decompensated cirrhosis $(1.3$; $95 \% \mathrm{Cl} 0.9-$ 1.7) ( Table 5). On multivariable analysis, every 1 point increase in the Elixhauser index (adjusted odds ratio [aOR], 1.1; $95 \% \mathrm{Cl} 1.1-1.3$ ) and biliary sphincterotomy (aOR 1.7; $95 \% \mathrm{Cl}$ $1.2-2.3$ ) increased the risk of PEP. Therapeutic ERCP was also associated with an increased risk of PEP (aOR 1.1; 95\% Cl 1.1 1.3). The use of a pancreatic stent (aOR $0.8 ; 95 \% \mathrm{Cl} 0.7-0.9$ ), ERCP for biliary indications (aOR $0.8 ; 95 \% \mathrm{Cl} 0.6-0.9$ ), male sex (aOR 0.8; $95 \% \mathrm{Cl} 0.7-0.9$ ), medium hospital size (aOR 0.8; $95 \% \mathrm{Cl} 0.7-0.9$ ), and large hospital size (aOR 0.6; $95 \% \mathrm{Cl} 0.4-$ $0.6)$ were associated with a decreased risk of PEP. 
- Table 2 Indications and outcomes of ERCP among cirrhotic patients and non-cirrhotic controls.

\begin{tabular}{|c|c|c|c|}
\hline & Cirrhosis $(n=3228), \%$ & No cirrhosis ( $n=12$ 912), \% & $P$ value \\
\hline \multicolumn{4}{|l|}{ Indications for ERCP } \\
\hline - CBD stone with or without gallstones & 73.4 & 75.9 & 0.51 \\
\hline - Cholangitis & 18.9 & 15.1 & $<0.01$ \\
\hline - Acute biliary pancreatitis & 26.6 & 33.8 & $<0.01$ \\
\hline - Biliary stricture/disease & 26.9 & 16.3 & $<0.01$ \\
\hline " Jaundice & 54.8 & 1.0 & $<0.01$ \\
\hline Diagnostic vs therapeutic indication for ERCP & & & $<0.01$ \\
\hline - Diagnostic ERCP & 33.2 & 24.6 & \\
\hline - Biliary indications & 78.5 & 82.6 & $<0.01$ \\
\hline - Pancreatic indications & 6.0 & 7.5 & 0.01 \\
\hline \multicolumn{4}{|l|}{ ERCP-related interventions } \\
\hline - Pancreatic stent & 5.5 & 6.9 & 0.01 \\
\hline - Biliary sphincterotomy and papillotomy & 57.8 & 68.6 & $<0.01$ \\
\hline
\end{tabular}

- Table 3 ERCP-related adverse events among cirrhotic inpatients and non-cirrhotic controls.

\begin{tabular}{|c|c|c|c|}
\hline & Cirrhosis $(n=3228)$ & No cirrhosis ( $n=12$ 912) & $P$ value \\
\hline \multicolumn{4}{|l|}{ ERCP-related adverse events, \% } \\
\hline - Post-ERCP pancreatitis & 12.0 & 10.4 & 0.01 \\
\hline - ERCP-associated hemorrhage & 2.1 & 1.2 & $<0.01$ \\
\hline - Perforation & 0.2 & 0.1 & 0.75 \\
\hline - Cholecystitis/cholangitis & 0.3 & 0.4 & 0.49 \\
\hline - Death & IS & IS & 0.87 \\
\hline \multicolumn{4}{|l|}{ Health services } \\
\hline - Length of stay, mean (SE), days & $9.1(0.2)$ & $6.5(0.1)$ & $<0.01$ \\
\hline - Total cost, mean (SE), \$ & $79282(631.2)$ & $57786(612.9)$ & $<0.01$ \\
\hline - In-hospital death, \% & 4.5 & 1.4 & $<0.01$ \\
\hline
\end{tabular}

\section{Multivariable analysis of post-ERCP bleeding}

On multivariable analysis, we adjusted for gender, hospital size, comorbidities, teaching hospital status, pancreatic stent placement, biliary sphincterotomy, and presence of sepsis for development of post-ERCP bleeding. The presence of decompensated cirrhosis (aOR 2.6; $95 \% \mathrm{Cl} 2.2-3.2$ ), compensated cirrhosis (aOR 2.2; $95 \% \mathrm{Cl} 1.2-3.9$ ), every 1 point increase in Elixhauser index (aOR 2.2; 95\%Cl 2.1-2.3), therapeutic ERCPs (aOR 1.4; $95 \% \mathrm{Cl} 1.2-2.1$ ), and biliary sphincterotomy (aOR 1.5; $95 \% \mathrm{Cl}$ $1.1-2.1$ ) independently increased the risk of post-ERCP bleed- ing. Performing ERCPs in medium (aOR 0.7 ; $95 \% \mathrm{Cl} 0.6-0.9$ ) and large hospitals (aOR 0.5; $95 \% \mathrm{Cl} 0.4-0.6$ ) was associated with a decreased risk of post-ERCP bleeding ( $>$ Table 6 ). $>$ Supplementary Tables $\mathbf{2}$ and $\mathbf{3}$ illustrate the impact of cirrhosis on the length of stay and hospitalization costs.

\section{Discussion}

In this large national study, we demonstrate that performing biliary sphincterotomy in both compensated and decompensated cirrhosis is independently associated with an increased risk 
- Table 4 ERCP-related adverse events among cirrhotic inpatients and non-cirrhotic controls.

\begin{tabular}{|c|c|c|c|}
\hline & Cirrhosis, \% & No cirrhosis, \% & $P$ value \\
\hline Diagnostic ERCP $(n=4251)$ & $(n=1073)$ & $(n=3178)$ & \\
\hline - Post-ERCP pancreatitis & 13.1 & 12.4 & 0.58 \\
\hline - ERCP-associated hemorrhage & 2.0 & 1.2 & 0.08 \\
\hline - Perforation & 0.3 & 0.1 & 0.07 \\
\hline - Cholecystitis/cholangitis & 0.3 & 0.7 & 0.11 \\
\hline - Death & IS & IS & 0.80 \\
\hline Therapeutic ERCP $(n=11889)$ & $(n=2155)$ & $(n=9734)$ & \\
\hline - Post-ERCP pancreatitis & 11.4 & 9.7 & 0.02 \\
\hline - ERCP-associated hemorrhage & 2.2 & 1.2 & $<0.01$ \\
\hline - Perforation & 0.1 & 0.2 & 0.50 \\
\hline - Cholecystitis/cholangitis & 0.4 & 0.3 & 0.75 \\
\hline - Death & is & IS & 0.81 \\
\hline Biliary ERCP $(n=13203)$ & $(n=2535)$ & $(n=10668)$ & \\
\hline - Post-ERCP pancreatitis & 11.7 & 9.4 & 0.01 \\
\hline - ERCP-associated hemorrhage & 2.3 & 1.3 & $<0.01$ \\
\hline - Perforation & 0.1 & 0.1 & 0.43 \\
\hline - Cholecystitis/cholangitis & 0.4 & 0.4 & 0.94 \\
\hline - Death & IS & IS & 0.82 \\
\hline Pancreatic ERCP $(n=1161)$ & $(n=192)$ & $(n=969)$ & \\
\hline - Post-ERCP pancreatitis & 25.5 & 30.0 & 0.21 \\
\hline - ERCP-associated hemorrhage & 2.1 & 1.1 & 0.29 \\
\hline - Perforation & 0.0 & 0.6 & 0.27 \\
\hline - Cholecystitis/cholangitis & 0.0 & 0.3 & 0.44 \\
\hline - Death & IS & IS & 0.90 \\
\hline
\end{tabular}

NOTE. According to the data user agreement, any individual table cell counts of 10 or fewer cannot be presented to preserve patient confidentiality. In such instances, data are suppressed. IS, information suppressed.

of post-procedure bleeding. Performing biliary sphincterotomy was associated with an increased risk of PEP, while placement of a pancreatic stent was associated with a decreased risk of PEP. Also, performing ERCPs in medium and large hospitals was associated with a decreased risk of post-ERCP bleeding and PEP. The increased risk of adverse event in cirrhotic patients was not observed in patients who underwent ERCP for purely diagnostic purposes. Therapeutic ERCP was associated with an increased risk of PEP and post-procedural bleeding.

Previously published studies have reported variable rates of bleeding after ERCP in patients with underlying cirrhosis [3, 5, $6]$. While two studies reported a higher risk of bleeding in cirrhotic patients compared to controls $[3,6]$, one study did not demonstrate any difference [5]. Among the two studies suggesting an increased risk of bleeding in cirrhotics, one reported a $25 \%$ rate of clinically significant bleeding after ERCP in patients with Child-Pugh Class C versus $3 \%$ in non-cirrhotic pa- tients [3]. In the other study, the rate of significant bleeding was reported as $3.8 \%$ [6]. A recently published study reported a bleeding rate of only $1.1 \%$ in cirrhotics [5]. In the present study, the rate of bleeding was $2.1 \%$ and was significantly higher in cirrhotic patients than non-cirrhotic patients. The discrepancy between this study and some of the earlier studies could be because of differences in the patient populations and type of procedures. For instance, in the study by Adler et al. [5], biliary sphincterotomy was performed in only $15 \%$ of the procedures ( 82 of the 538 procedures), while $57.8 \%$ of the patients in our cohort underwent biliary sphincterotomy. We also observed that the risk of post-procedure bleeding was higher with compensated cirrhosis. This may be related to the fact that, even in early well compensated cirrhosis, platelet dysfunction may be evident secondary to portal hypertension. This may also explain the low risk of adverse events in patients who underwent ERCP for purely diagnostic purposes. 
- Table 5 Multivariable analysis of predictors of post-ERCP pancreatitis.

Adjusted odds ratio ( $95 \%$ confidence interval)

Liver disease status

\begin{tabular}{|c|c|c|}
\hline - No cirrhosis & Ref & \\
\hline - Compensated cirrhosis & $1.0(0.6-1.2)$ & 0.10 \\
\hline - Decompensated cirrhosis & $1.3(0.9-1.7)$ & 0.22 \\
\hline \multicolumn{3}{|l|}{ Cirrhosis type } \\
\hline - Non-alcoholic & Ref & \\
\hline - Alcoholic & $1.7(1.3-2.3)$ & $<0.01$ \\
\hline - Age, years & $0.9(0.9-1.01)$ & 0.19 \\
\hline Elixhauser index (every 1 point increase) & $1.1(1.1-1.3)$ & $<0.01$ \\
\hline \multicolumn{3}{|l|}{ Sex } \\
\hline - Female & Ref & \\
\hline - Male & $0.8(0.7-0.9)$ & $<0.01$ \\
\hline \multicolumn{3}{|l|}{ Hospital teaching status } \\
\hline - Nonteaching & Ref & \\
\hline - Teaching & $1.2(0.8-1.5)$ & 0.22 \\
\hline \multicolumn{3}{|l|}{ Hospital size } \\
\hline - Small & Ref & \\
\hline - Medium & $0.8(0.8-0.9)$ & 0.03 \\
\hline - Large & $0.6(0.4-0.6)$ & 0.01 \\
\hline \multicolumn{3}{|l|}{ ERCP-related interventions } \\
\hline - Pancreatic stent & $0.8(0.7-0.9)$ & $<0.01$ \\
\hline - Biliary sphincterotomy & $1.7(1.2-2.3)$ & $<0.01$ \\
\hline \multicolumn{3}{|l|}{ ERCP-type } \\
\hline - Diagnostic ERCP & Ref & \\
\hline - Therapeutic ERCP & $1.1(1.1-1.3)$ & $<0.01$ \\
\hline \multicolumn{3}{|l|}{ ERCP-indication } \\
\hline - Pancreatic & Ref & \\
\hline - Biliary & $0.8(0.6-0.9)$ & 0.02 \\
\hline
\end{tabular}

Previous meta-analyses have shown that sphincterotomy, coagulopathy, use of anticoagulants within 72 hours of sphincterotomy, precut sphincterotomy, and low case volume of the endoscopist were associated with post-ERCP bleeding [1315]. We observed that performing ERCPs in medium and large size hospitals decreased the risk of post-procedure bleeding. Better experience of the endoscopists in managing complex cirrhotic patients, a larger caseload, and appropriate infrastructure to deliver high-quality outcomes in a larger center may explain the improved outcomes.

Although on univariate analysis, we observed an increase in the risk of PEP in cirrhotic patients, after adjusting for other factors that increase pancreatitis risk, the presence of cirrhosis did not increase the risk of PEP. It was interesting to observe the higher rate of PEP in patients with alcoholic cirrhosis compared to non-alcoholic cirrhotic patients. The 2014 European Society of Gastrointestinal Endoscopy prophylaxis of PEP guidelines has suggested that there is no evidence to support the impact of hospital ERCP volume on PEP [16]. A previous study using the NIS database has reported that performing ERCP at high-volume hospitals had lower procedural failure rates [17]. Our data indicate that performing ERCPs in medium and large hospitals is associated with a decreased risk of PEP.

We did not find a significant difference in the risk of postERCP infections in patients with and without cirrhosis. Infectious adverse events were very uncommon in our cohort with an incidence of $0.3 \%$. This risk is less than that reported in a recently published study where infections occurred in $16 / 530$ 
Table 6 Multivariable analysis of predictors of post-ERCP bleeding.

\begin{tabular}{|c|c|c|}
\hline & Adjusted odds ratio ( $95 \%$ confidence interval) & $P$ value \\
\hline \multicolumn{3}{|l|}{ Liver disease status } \\
\hline - No cirrhosis & Ref & \\
\hline - Compensated cirrhosis & $2.2(1.2-3.9)$ & $<0.01$ \\
\hline - Decompensated cirrhosis & $2.6(2.2-3.2)$ & $<0.01$ \\
\hline \multicolumn{3}{|l|}{ Cirrhosis type } \\
\hline - Non-alcoholic & Ref & \\
\hline - Alcoholic & $0.5(0.2-1.4)$ & 0.16 \\
\hline Age, years & $1.01(1.0-1.02)$ & 0.01 \\
\hline Elixhauser index (every 1 point increase) & $2.2(2.1-2.3)$ & $<0.01$ \\
\hline \multicolumn{3}{|l|}{ Sex } \\
\hline - Female & Ref & \\
\hline - Male & $0.9(0.7-1.2)$ & 0.38 \\
\hline \multicolumn{3}{|l|}{ Hospital teaching status } \\
\hline - Nonteaching & Ref & \\
\hline - Teaching & $0.9(0.8-1.2)$ & 0.32 \\
\hline \multicolumn{3}{|l|}{ Hospital size } \\
\hline - Small & Ref & \\
\hline - Medium & $0.7(0.6-0.9)$ & 0.04 \\
\hline - Large & $0.5(0.4-0.6)$ & 0.01 \\
\hline \multicolumn{3}{|l|}{ ERCP-related interventions } \\
\hline - Pancreatic stent & $1.1(0.7-1.9)$ & 0.93 \\
\hline - Biliary sphincterotomy & $1.5(1.1-2.1)$ & $<0.01$ \\
\hline \multicolumn{3}{|l|}{ ERCP-type } \\
\hline - Diagnostic ERCP & Ref & \\
\hline - Therapeutic ERCP & $1.4(1.2-2.1)$ & $<0.01$ \\
\hline \multicolumn{3}{|l|}{ ERCP-indication } \\
\hline - Pancreatic & Ref & \\
\hline - Biliary & $1.5(0.8-2.6)$ & 0.21 \\
\hline
\end{tabular}

procedures (3\%) [5]. Most patients with cirrhosis are on antibiotics for spontaneous bacterial peritonitis prophylaxis and all patients with recent variceal bleed are also on antibiotics. This may explain the low risk of infections in patients with cirrhosis. Our data complement other studies reporting an increased PEP risk with biliary sphincterotomy and a decreased PEP risk by using prophylactic pancreatic stenting [16].

We also observed that decompensated liver cirrhosis is associated with increased length of stay and hospitalization costs after ERCP. This is likely related to an associated increased risk of adverse events when ERCP was performed on patients with underlying cirrhosis. We also observed that an increase in the
Elixhauser index was associated with a decreased length of stay. It is likely that sicker patients are more likely to die in the hospital resulting in decreased length of stay. This is reflected in the higher hospitalization costs for patients with higher Elixhauser index. We also observed a decreased length of hospital stay in patients admitted to teaching hospitals compared to non-teaching hospitals. The availability of appropriate infrastructure with multidisciplinary support to deliver high-quality outcomes may explain the lower length of stay in teaching hospitals.

Our study had several limitations. The diagnostic codes and identification of comorbidities are dependent on the accuracy 
of coding procedures. Although we did not validate the diagnoses, validation is routinely performed by the Agency for Healthcare Research and Quality [Agency for Healthcare Research and Quality Web site. http://www.hcup-us.ahrq.gov/nisoverview. jsp; accessed 30 September 2015]. In addition, we could not study whether the presence of primary sclerosing cholangitis (PSC) impacted the risk of post-ERCP adverse events. The code used for PSC is not accurate in these databases which limited our ability to investigate this subset of patients [18]. Moreover, the database reports only inpatients with cirrhosis who undergo ERCP. The NIS database does not provide information on use of pharmacological prophylaxis such as rectal indomethacin or laboratory values used to calculate the model for end-stage liver disease score or the platelet count and the internationalized normalized ratio which may impact the outcome, in particular bleeding risk in patients. Additionally, we observed a high proportion of patients (26\%) who underwent ERCPs for purely diagnostic indications in our cohort. This may reflect practice pattern differences in smaller hospitals where the lack of a dedicated radiologist or lack of endosonographers could explain the increased use of ERCPs for diagnostic indications. We also observed that alcoholic cirrhosis constituted only $10 \%$ of the cirrhotic population. Errors in coding could explain both of these observations. Finally, in our study, we included all cirrhosis patients but part of non-cirrhosis patients randomly selected from the NIS data. The variances of the non-cirrhosis cohort might be underestimated because we ignored an extra sampling error of this cohort. However, this sampling error was actually rather small given the large sample size of this non-cirrhosis cohort (over 12900 patients).

Despite these limitations, this study has several strengths and clinical implications. Use of data from a nationwide representative sample increases the generalizability of the results and minimizes the possible biases that may be seen with single-center studies. To our knowledge, this is the first population-based study from the USA to assess the safety of ERCP in patients with liver cirrhosis. Overall, ERCP can be performed safely in cirrhotic patients with the caveat that it may increase the risk of post-procedure bleeding. Performing biliary sphincterotomy judiciously and referral of cirrhotic patients for ERCPs to large hospitals may improve outcomes.

\section{Competing interests}

Udayakumar Navaneethan is a consultant for AbbVie, Janssen and Takeda. Shyam Varadarajulu is a consultant for Boston Scientific and Olympus. Mansour Parsi is a consultant for Boston Scientific. None of the other authors declared a financial conflict of interest.

\section{References}

[1] NIH state-of-the-science statement on endoscopic retrograde cholangiopancreatography (ERCP) for diagnosis and therapy. NIH Consens State Sci Statements 2002; 19: 1-26

[2] Adler DG, Baron TH, Davila RE et al. Standards of Practice Committee of American Society for Gastrointestinal Endoscopy. ASGE guideline: the role of ERCP in diseases of the biliary tract and the pancreas. Gastrointest Endosc 2005; 62: 1-8

[3] Prat F, Tennenbaum R, Ponsot P et al. Endoscopic sphincterotomy in patients with liver cirrhosis. Gastrointest Endosc 1996; 43: 127-131

[4] Mosko JD, Nguyen GC. Increased perioperative mortality following bariatric surgery among patients with cirrhosis. Clin Gastroenterol Hepatol 2011; 9: 897-901

[5] Adler DG, Haseeb A, Francis G et al. Efficacy and safety of therapeutic ERCP in patients with cirrhosis: a large multicenter study. Gastrointest Endosc 2016; 83: $353-359$

[6] Li DM, Zhao J, Zhao Q et al. Safety and efficacy of endoscopic retrograde cholangiopancreatography for common bile duct stones in liver cirrhotic patients. J Huazhong Univ Sci Technol Med Sci 2014; 34: $612-615$

[7] de Franchis R. Evolving consensus in portal hypertension. Report of the Baveno IV consensus workshop on methodology of diagnosis and therapy in portal hypertension. J Hepatol 2005; 43: 167-176

[8] Yadav D, O'Connell M, Papachristou GI. Natural history following the first attack of acute pancreatitis. Am J Gastroenterol 2012; 107 : $1096-1103$

[9] Inamdar S, Berzin TM, Sejpal DV et al. Pregnancy is a risk factor for pancreatitis after endoscopic retrograde cholangiopancreatography in a national cohort study. Clin Gastroenterol Hepatol 2016; 14: $107-$ 114

[10] Elixhauser A, Steiner C, Harris DR et al. Comorbidity measures for use with administrative data. Med Care 1998; 36: 8-27

[11] Deyo RA, Cherkin DC, Ciol MA. Adapting a clinical comorbidity index for use with ICD-9-CM administrative databases. J Clin Epidemiol 1992; 45: 613-619

[12] Southern DA, Quan H, Ghali WA. Comparison of the Elixhauser and Charlson/Deyo methods of comorbidity measurement in administrative data. Med Care 2004; 42: 355-360

[13] Masci E, Toti G, Mariani A et al. Complications of diagnostic and therapeutic ERCP: a prospective multicenter study. Am J Gastroenterol 2001; 96: 417-423

[14] Loperfido S, Angelini G, Benedetti G et al. Major early complications from diagnostic and therapeutic ERCP: a prospective multicenter study. Gastrointest Endosc 1998; 48: 1- 10

[15] Andriulli A, Loperfido S, Napolitano G et al. Incidence rates of postERCP complications: a systematic survey of prospective studies. Am J Gastroenterol 2007; 102: $1781-1788$

[16] Dumonceau J-M, Andriulli A, Elmunzer B] et al. Prophylaxis of postERCP pancreatitis: European Society of Gastrointestinal Endoscopy (ESGE) guideline - updated June 2014. Endoscopy 2014; 46: 799815

[17] Varadarajulu S, Kilgore ML, Wilcox CM et al. Relationship among hospital ERCP volume, length of stay, and technical outcomes. Gastrointest Endosc 2006; 64: 338 - 347

[18] Molodecky NA, Myers RP, Barkema HW et al. Validity of administrative data for the diagnosis of primary sclerosing cholangitis: a populationbased study. Liver Int 2011; 31: 712-720 
- Appendix 1 ERCP ICD-9 procedure codes used for inclusion of patients in the study.

\begin{tabular}{|c|c|}
\hline $\begin{array}{l}571.2,571.5 \text { and } 571.6 \\
571.2 \\
571.5 \\
571.6\end{array}$ & $\begin{array}{l}\text { Cirrhosis } \\
\text { Alcoholic cirrhosis } \\
\text { Cirrhosis from all others other than alcohol } \\
\text { Biliary cirrhosis }\end{array}$ \\
\hline Procedure codes & Procedure description \\
\hline \multicolumn{2}{|l|}{ Diagnostic ERCP codes } \\
\hline 51.10 & Endoscopic retrograde cholangiopancreatography [ERCP] \\
\hline 51.11 & Endoscopic retrograde cholangiography [ERC] \\
\hline 51.14 & Other closed [endoscopic] biopsy of biliary duct or sphincter of Oddi \\
\hline 52.13 & Endoscopic retrograde pancreatography [ERP] \\
\hline 52.14 & Closed [endoscopic] biopsy of pancreatic duct \\
\hline \multicolumn{2}{|l|}{ Therapeutic ERCP codes } \\
\hline 51.84 & Endoscopic dilation of ampulla and biliary duct \\
\hline 51.85 & Endoscopic sphincterotomy and papillotomy \\
\hline 51.86 & Endoscopic insertion of nasobiliary drainage tube \\
\hline 51.87 & Endoscopic insertion of stent (tube) into bile duct \\
\hline 51.88 & Endoscopic removal of stone(s) from biliary tract \\
\hline 52.93 & $\begin{array}{l}\text { Endoscopic insertion of stent (tube) into } \\
\text { pancreatic duct }\end{array}$ \\
\hline 52.94 & Endoscopic removal of stone(s) from pancreatic duct \\
\hline 52.97 & Endoscopic insertion of nasopancreatic drainage tube \\
\hline 52.98 & Endoscopic dilation of pancreatic duct \\
\hline
\end{tabular}

- Appendix 2 Bed size categories, by region.

\section{Location and teaching status}

\section{Hospital bed size}

\begin{tabular}{|l|l|l} 
Small & Medium & Large \\
\hline
\end{tabular}

\section{Northeast}

. Rural

- Urban, non-teaching

- Urban, teaching

$1-49$

$50-99$

$100+$

Midwest

- Rural

- Urban, non-teaching

- Urban, teaching

$1-49$

125-199

$200+$

$1-124$

$250-424$

$425+$

$1-249$

$1-29$

$1-74$

$1-249$

$30-49$

$50+$

$75-174$

$175+$

- Urban, teaching

South

- Rural

$1-39$

$250-374$

$375+$

- Urban, non-teaching

$1-99$

- Urban, teaching

$1-249$

$40-74$

$75+$

$200+$

$100-199$

$450+$

West

- Rural

- Urban, non-teaching

- Urban, teaching

hing


- Supplementary Table 1 ERCP related adverse events among alcohol-related cirrhosis vs non-alcohol related cirrhosis.

\begin{tabular}{|c|c|c|c|}
\hline & Alcoholic cirrhosis $(n=332)$ & Non-alcoholic cirrhosis $(n=2896)$ & $P$ value \\
\hline \multicolumn{4}{|l|}{ ERCP-related adverse events, \% } \\
\hline - Post-ERCP pancreatitis & 20.2 & 11.0 & $<0.01$ \\
\hline - ERCP-associated hemorrhage & 1.2 & 2.2 & 0.22 \\
\hline - Perforation & 0.1 & 0.2 & 0.45 \\
\hline - Cholecystitis/cholangitis & 0.3 & 0.4 & 0.34 \\
\hline - Death & IS & IS & 0.88 \\
\hline \multicolumn{4}{|l|}{ Health services } \\
\hline - Length of stay, mean (SE), days & $10.6(0.7)$ & $7.0(0.1)$ & $<0.01$ \\
\hline - Total cost, mean (SE), \$ & $90910(6345)$ & $61426(627)$ & $<0.01$ \\
\hline . In-hospital death, \% & 3.3 & 4.3 & 0.40 \\
\hline \multicolumn{4}{|c|}{$\begin{array}{l}\text { SE, standard error. } \\
\text { NOTE. According to the data user agreement, any individual table cell counts of } 10 \text { or fewer cannot be presented to preserve patient confidentiality. In such instan- } \\
\text { ces, data are suppressed. IS, information suppressed. }\end{array}$} \\
\hline
\end{tabular}

Supplementary Table 2 Multivariate analysis of factors associated with length of stay.

\begin{tabular}{|c|c|c|}
\hline & Adjusted odds ratio (95\% confidence interval) & $P$ value \\
\hline \multicolumn{3}{|l|}{ Liver disease status } \\
\hline - No cirrhosis & Ref & \\
\hline - Compensated cirrhosis & $2.44(0.35-4.52)$ & 0.02 \\
\hline - Decompensated cirrhosis & $5.23(2.61-7.86)$ & $<0.001$ \\
\hline Age, years & $0.00(-0.02$ to -0.02$)$ & 0.97 \\
\hline Elixhauser index (every 1 increase) & $0.29(0.15-0.44)$ & $<0.001$ \\
\hline \multicolumn{3}{|l|}{ Sex } \\
\hline - Male & Ref & \\
\hline - Female & $-0.44(-1.17$ to -0.29$)$ & 0.24 \\
\hline \multicolumn{3}{|l|}{ Hospital teaching status } \\
\hline - Nonteaching & Ref & \\
\hline - Teaching & $0.00(0.00-0.00)$ & 0.007 \\
\hline Pancreatic stent & $1.58(-5.62$ to 8.79$)$ & 0.67 \\
\hline Biliary sphincterotomy & $-0.87(-1.88$ to -0.14$)$ & 0.09 \\
\hline
\end{tabular}


- Supplementary Table 3 Multivariate analysis of factors associated with hospitalization costs.

\begin{tabular}{|c|c|c|}
\hline & Adjusted odds ratio ( $95 \%$ confidence interval) & $P$ value \\
\hline \multicolumn{3}{|l|}{ Liver disease status } \\
\hline - No cirrhosis & Ref & \\
\hline - Compensated cirrhosis & $150.52(-14076.71$ to 14377.75$)$ & 0.98 \\
\hline - Decompensated cirrhosis & 33917.23 (15 956.66-51 877.79) & $<0.001$ \\
\hline Age & $35.78(-132.18$ to 203.74$)$ & 0.68 \\
\hline Elixhauser index (every 1 point increase) & $1778.19(798.48-2757.92)$ & $<0.001$ \\
\hline \multicolumn{3}{|l|}{ Sex } \\
\hline - Male & Ref & \\
\hline - Female & $-2623.73(-7605.74$ to -2358.28$)$ & 0.30 \\
\hline \multicolumn{3}{|l|}{ Hospital teaching status } \\
\hline - Nonteaching & Ref & \\
\hline - Teaching & $0.20(-0.05$ to 0.45$)$ & 0.12 \\
\hline \multicolumn{3}{|l|}{ ERCP-related interventions } \\
\hline - Pancreatic stent & $2233.22(-47000.91$ to 51467.36$)$ & 0.93 \\
\hline - Biliary sphincterotomy & $-3487.95(-10400.02$ to -3424.13$)$ & 0.32 \\
\hline
\end{tabular}

